\title{
Lasmiditan for acute treatment of migraine in patients with cardiovascular risk factors: post-hoc analysis of pooled results from 2 randomized, double-blind, placebo- controlled, phase 3 trials
}

Robert E. Shapiro ${ }^{1}$, Helen M. Hochstetler ${ }^{2^{*}}$ (1), Ellen B. Dennehy²,3, Rashna Khanna², Erin Gautier Doty ${ }^{2}$, Paul H. Berg ${ }^{2}$ and Amaal J. Starling ${ }^{4}$

\begin{abstract}
Background: In addition to the increased risk for cardiovascular (CV) disease and CV events associated with migraine, patients with migraine can also present with a number of CV risk factors (CVRFs). Existing treatment options can be limited due to contraindications, increased burden associated with monitoring, or patient avoidance of side effects. Safe and effective migraine treatment options are needed for patients with migraine and a history of CV or cerebrovascular disease or with increased risk for CV events. This analysis was designed to evaluate the safety and efficacy of oral lasmiditan, a selective serotonin 5-hydroxytryptamine $1 \mathrm{~F}$ receptor agonist, in acute treatment of migraine attacks in patients with CVRFs.

Methods: SAMURAI and SPARTAN were similarly designed, Phase 3, randomized, double-blind, placebo-controlled trials in adults treating a single migraine attack with lasmiditan 50, 100, or $200 \mathrm{mg}$. Both studies included patients with CVRFs, and SPARTAN allowed patients with coronary artery disease, clinically significant arrhythmia, or uncontrolled hypertension. Efficacy and safety of lasmiditan in subgroups of patients with differing levels of CVRFs are reported. For efficacy analyses, logistic regression was used to assess treatment-by-subgroup interactions. For safety analyses, Cochran-Mantel-Haenszel test of general association evaluated treatment comparisons; Mantel-Haenszel odds ratio assessed significant treatment effects.

Results: In this pooled analysis, a total of 4439 patients received $\geq 1$ dose of study drug. A total of 3500 patients (78.8\%) had $\geq 1$ CVRF, and 1833 patients (41.3\%) had $\geq 2$ CVRFs at baseline. Both trials met the primary endpoints of headache pain freedom and most bothersome symptom freedom at $2 \mathrm{~h}$. The presence of CVRFs did not affect efficacy results. There was a low frequency of likely CV treatment-emergent adverse events (TEAEs) overall (lasmiditan, 30 [0.9\%]; placebo, 5 [0.4\%]). There was no statistical difference in the frequency of likely CV TEAEs in either the absence or presence of any CVRFs. The only likely CV TEAE seen across patients with $\geq 1, \geq 2, \geq 3$, or $\geq 4$ CVRFs was palpitations.

\footnotetext{
* Correspondence: hochstetler_helen_m@lilly.com

${ }^{2}$ Eli Lilly and Company, and/or one of its subsidiaries, Lilly Corporate Center,

Indianapolis, IN 46285, USA

Full list of author information is available at the end of the article
}

(c) The Author(s). 2019 Open Access This article is distributed under the terms of the Creative Commons Attribution 4.0 International License (http://creativecommons.org/licenses/by/4.0/), which permits unrestricted use, distribution, and reproduction in any medium, provided you give appropriate credit to the original author(s) and the source, provide a link to the Creative Commons license, and indicate if changes were made. 
(Continued from previous page)

Conclusions: When analyzed by the presence of CVRFs, there was no statistical difference in lasmiditan efficacy or the frequency of likely CV TEAEs. Despite the analysis being limited by a single-migraine-attack design, the lack of differences in efficacy and safety with increasing numbers of CVRFs indicates that lasmiditan might be considered in the treatment algorithm for patients with CVRFs. Future studies are needed to assess long-term efficacy and safety.

Trial registration: ClinicalTrials.gov NCT02439320 (SAMURAI), registered 18 March 2015 and ClinicalTrials.gov NCT026 05174 (SPARTAN), registered 11 November 2015.

Keywords: Migraine, Cardiovascular disease, Lasmiditan, Safety, Ditan,

\section{Background}

Migraine is a neurologic disease characterized by severe, intermittent headache attacks with associated symptoms including nausea, vomiting, phonophobia, and photophobia that can be chronic and disabling [1]. The disease can interfere significantly with occupational, educational, household, family, and social responsibilities [2]. It is the second largest cause of years lost to disability [3].

Migraine is an independent risk factor for cardiovascular (CV) disease $[4,5]$ and is associated with a number of $\mathrm{CV}$ events, including ischemic stroke, transient ischemic attack, ischemic heart disease, and myocardial infarction, as well as increased morbidity and mortality [6-11]. Although both migraine with and without aura are known to be associated with CV disease, these associations are more significant in patients with migraine with aura [5, 11-13]. A recent meta-analysis demonstrated that the presence of aura significantly affects the risk of stroke (adjusted hazard ratio [aHR] aura 1.56; $95 \%$ confidence interval [CI] $1.30-1.87$ vs. aHR no aura $1.11 ; 95 \%$ CI $\left.0.94-1.31 ; \quad p_{\text {interaction }}=0.01\right) \quad$ [5]. In addition, statistical heterogeneity was lower for all CV and cerebrovascular outcomes when results were stratified by the presence of aura [5]. Existing options for acute migraine treatment may be contraindicated in patients with CV history or risk [14, 15]. For example, nonsteroidal anti-inflammatory drugs are associated with an increased risk of $\mathrm{CV}$, thrombotic, and upper gastrointestinal events [16-18]. Due to vasoconstriction associated with the 5-hydroxytryptamine receptor $1 \mathrm{~B}$ $\left(5-\mathrm{HT}_{1 \mathrm{~B}}\right)$ activity, triptans are contraindicated in patients with ischemic coronary artery disease (CAD), coronary artery vasospasm, Wolff-Parkinson-White syndrome, peripheral vascular disease, ischemic bowel disease, and uncontrolled hypertension and in patients with a history of cerebrovascular ischemic events [1925]. Approximately 2 million women and 665,000 men in the United States have episodic migraine and a history of $\geq 1 \mathrm{CV}$ event, condition, or procedure that may limit the use of triptans [26].

The desire to discover effective migraine treatments without vasoconstrictive properties led to the development of selective 5-hydroxytryptamine receptor $1 \mathrm{~F}\left(5-\mathrm{HT}_{1 \mathrm{~F}}\right)$ agonists and other molecules [27-33]. Lasmiditan selectively targets $5-\mathrm{HT}_{1 \mathrm{~F}}$ receptors on neurons in the central and peripheral trigeminal system, decreasing neuropeptide release and inhibiting pain pathways, including the trigeminal nerve $[34,35]$. Preclinical studies have demonstrated that messenger RNA for $5-\mathrm{HT}_{1 \mathrm{~F}}$ receptors is highly expressed in human middle cerebral arteries [36] and human coronary arteries [37], but in vitro studies suggest that $5-\mathrm{HT}_{1 \mathrm{~F}}$ receptors do not mediate significant vasoconstriction effects in human cerebral or coronary vessels [37-40]. Data from nonclinical animal and in vitro studies indicate that lasmiditan does not cause vasoconstriction in coronary, carotid, and internal mammary arteries [35, 41, 42].

The efficacy and safety of oral lasmiditan in the acute treatment of migraine attacks have been demonstrated in 2 randomized, double-blind, placebo-controlled, Phase 3 studies, SAMURAI and SPARTAN [43, 44]. This publication reports the pooled safety and efficacy of lasmiditan in a subpopulation of patients with CV risk factors (CVRFs).

\section{Methods}

\section{Patients and study design}

Detailed design and clinical results of SAMURAI and SPARTAN have been reported $[43,44]$. SAMURAI and SPARTAN shared many study design elements, allowing for integrated analyses. Briefly, both trials were randomized, double-blind, placebo-controlled, Phase 3 studies of a single migraine attack. These studies were conducted in accordance with the principles of the Declaration of Helsinki. The institutional review board or independent ethics committee at each site approved the protocols, and all patients provided written informed consent. All authors had access to the study data and have reviewed and approved the final manuscript. SAMURAI and SPARTAN were conducted in patients with migraine with and without aura (based on history alone), with the primary objective of evaluating the efficacy of lasmiditan versus placebo as measured by the proportion of patients who became headache pain-free and most bothersome symptom (MBS)-free at $2 \mathrm{~h}$. Patients identified their MBS from nausea, photophobia, or phonophobia at baseline. Patients were randomized to a double- 
blind, 2-dose sequence of oral lasmiditan $200 \mathrm{mg}, 100 \mathrm{mg}$, or $50 \mathrm{mg}$ (SPARTAN only) or placebo (in equal proportions for the first dose); patients were allowed to take a second dose of study drug of the same strength 2 to $24 \mathrm{~h}$ after the first dose if symptoms persisted or returned. For the second dose, the placebo arm received placebo and active treatment arms received either the same strength of lasmiditan or placebo (2:1 ratio).

\section{Inclusion/exclusion criteria}

The 2 trials enrolled very similar populations. However, SPARTAN allowed enrollment of patients with known CAD, clinically significant arrhythmia, or uncontrolled hypertension, whereas such patients were excluded in SAMURAI.

\section{Baseline cardiovascular/cerebrovascular-related history}

A patient was identified as having baseline $\mathrm{CV} /$ cerebrovascular-related history (CCRH) if the patient self-reported 1 or more conditions included in the narrow search terms of the following Standardized Medical Dictionary for Drug Regulatory Activities (MedDRA) Queries (SMQs): Cardiac arrhythmias, Cardiac failure, Cardiomyopathy, Central nervous system (CNS) vascular disorders, Embolic and thrombotic events, Hypertension, Ischemic heart disease, Pulmonary hypertension, and Torsade de pointes/QT prolongation.

\section{Concomitant cardiovascular medications}

CV medications were identified using the World Health Organization's Anatomical Therapeutic Chemical/Defined Daily Dose codes within the "Cardiac System" and "Antithrombotic Agents." The selected medications were then reviewed to confirm that the indication for use was a $\mathrm{CV}$ condition (per medical history or adverse event $[\mathrm{AE}]$ ). For example, $\mathrm{CV}$ medications being used for migraine prevention (eg, beta blockers and calcium channel blockers) as the indication were removed.

\section{Identification of cardiovascular risk factors}

For the pooled analyses of the primary objectives and safety measures, CVRFs of interest included the 6 variables that the American College of Cardiology/ American Heart Association Task Force on Practice Guidelines concluded were the most robust variables for prediction of a first CV event [45]. A present/absent criterion was applied to each variable to assess the proportion of patients with each potential risk. The variables and their defined thresholds were as follows: age $>40$ years [45], self-report of diabetes diagnosis, current smoker, baseline total cholesterol $\geq 240 \mathrm{mg} / \mathrm{dL}$ [45], baseline high-density lipoprotein cholesterol $<40 \mathrm{mg} / \mathrm{dL}$ for men or $<50 \mathrm{mg} / \mathrm{dL}$ for women [46], and baseline systolic blood pressure $\geq$
$140 \mathrm{mmHg}$ [47] and/or self-reported medical history of high blood pressure at baseline.

Subgroup efficacy analyses compared patients with $\geq 2$ CVRFs to those with 0 or $1 \mathrm{CVRF}(\mathrm{s})$, since many patients accrued 1 risk factor based on the age variable alone. Analyses of safety measures were performed based on the number of CVRFs, with categories of $0, \geq$ $1, \geq 2, \geq 3$, and $\geq 4$ risk factors.

\section{Study evaluations and analyses}

For efficacy evaluations, the proportions of patients achieving headache pain freedom and MBS freedom at $2 \mathrm{~h}$ after the first dose were compared in lasmiditan- and placebo-treated groups.

For safety evaluations, treatment-emergent adverse events (TEAEs), defined as events that initially occurred or worsened in severity after the first dose of study drug and occurred within $48 \mathrm{~h}$ of dose, were analyzed. AEs irrespective of temporal association with dosing were also analyzed because some CV events may have been identified at a later time (eg, during laboratory, vital signs, and/or electrocardiogram [ECG] assessments). Dose groups in the tables show the dose that the patients were randomized to; if the patient took a second dose of lasmiditan, their total dose may have been higher.

Potential CV AEs were identified by querying the full list of AEs for specific terms within the following SMQs: Cardiac arrhythmias, Cardiac failure, Cardiomyopathy, CNS vascular disorders, Embolic and thrombotic events, Hypertension, Ischemic heart disease, Pulmonary hypertension, and Torsade de pointes/QT prolongation along with the Preferred Terms (PTs) abdominal pain, abdominal pain upper, and abdominal pain lower. SMQs are validated, predetermined sets of MedDRA terms grouped together to aid with safety analyses and reporting. SMQs are independent of each other, and some terms could overlap between SMQs.

The resultant listing of potential CV AEs was then reviewed by a group of unblinded Eli Lilly physicians to determine which were likely $\mathrm{CV}$ in nature. For example, if an AE of "edema" occurred in close association with a local injury, then it was not considered a likely CV AE. The events determined to represent likely $\mathrm{CV}$ events (AEs and TEAEs) are discussed in detail.

\section{Statistical analysis}

Data handling rules and full analysis methods were previously described in Kuca et al. [43] and Wietecha et al. [44]. Efficacy analyses were conducted in the modified Intent-to-Treat population consisting of patients who took study drug within $4 \mathrm{~h}$ of migraine attack onset and had at least 1 postdose efficacy assessment. Safety and tolerability analyses were conducted in the Safety population consisting of patients who took study drug. For 
the analyses of headache pain freedom and MBS freedom in subgroups by number of CVRFs, the $p$ value was calculated for treatment-by-subgroup interaction, based on logistic regression with terms for study, subgroup, treatment, and treatment-by-subgroup in the model.

AEs were classified based on MedDRA version 21.0. Missing dates and times for dosing and AEs were imputed to avoid underestimation of frequency or duration of AEs and to increase the sensitivity of identifying TEAEs. The number and percentage of patients who reported TEAEs were summarized, and the results are presented by decreasing frequency of PTs in the all lasmiditan dose group.

Statistical comparisons were made between all lasmiditan doses combined and placebo as follows, unless otherwise noted. First, the Cochran-Mantel-Haenszel test of general association stratified by study was used for treatment comparisons of percentages. In addition to the Cochran-Mantel-Haenszel test, the Mantel-Haenszel odds ratio (OR) and the flag of $p$ value $<0.1$ Breslow-Day test for homogeneity of OR are displayed. ORs were created with treatment as the numerator and placebo as the denominator. In addition, study size-adjusted percentages are provided using the methodology of Crowe et al. [48].

Tests with 2 -sided $p$ values less than 0.05 are referred to as having statistical significance for a treatment difference, unless otherwise noted. However, $p$ values should not be overinterpreted for safety analyses. Except for prespecified hypotheses, they correspond to data-driven hypotheses and, hence, are only useful as a flagging mechanism.

\section{Results}

\section{Baseline patient characteristics}

Across both trials, a total of 4439 patients took $\geq 1$ dose of study drug, and 3701 patients were in the modified Intent-to-Treat population. The proportion of each CVRF is reported in Table 1. The distribution of the CVRFs (across all 6 factors) was balanced between the lasmiditan- and placebo-treated patients and across the lasmiditan dose groups (data not shown). The majority of patients $(78.8 \%)$ had $\geq 1$ CVRF (Table 1) with similar results between lasmiditan- and placebo-treated patients (Table 2). Overall, the frequencies of patients with $0,1,2,3,4$, or 5 CVRFs were balanced across the lasmiditan- and placebo-treated groups (Table 2). There were no patients with 6 CVRFs. Baseline CCRH was reported in $20.4 \%$ of patients. The most frequently reported types of CCRH were hypertension $(n=719)$, angina pectoris $(n=24)$, deep vein thrombosis $(n=16)$, myocardial infarction $(n=14)$, pulmonary embolism $(n=14)$, transient ischemic attack $(n=13)$, and CAD $(n=13)$. A small number of patients had contraindications to the use of a trip$\tan (n=15$ in SAMURAI; $n=75$ in SPARTAN).
A total of $21.8 \%$ of patients reported concomitant use of $\mathrm{CV}$ medication for reasons other than migraine at baseline. There was no statistical difference between treatment groups in the proportions of patients taking concomitant $\mathrm{CV}$ medicines either overall or for any medication class. The most commonly used medications in either of the treatment groups were agents acting on the renin-angiotensin system ( $n=393)$, lipid-modifying agents $(n=386)$, and beta-blocking agents $(n=231)$. Examples of other medications used were diuretics, antithrombotics, calcium channel blockers, and other cardiac therapies such as cardiac glycosides and antiarrhythmics.

\section{Efficacy}

Both studies met the primary objective, with significantly more lasmiditan-treated patients headache pain-free as well as MBS-free at $2 \mathrm{~h}$ at all doses compared with placebo-treated patients $[43,44]$. Within pooled subgroups of patients with 0 or $1 \mathrm{CVRF}(\mathrm{s})$ and with $\geq 2$ CVRFs, the proportion of patients who were pain-free and MBS-free at $2 \mathrm{~h}$ were unaffected by the degree of $\mathrm{CV}$ risk (Fig. 1).

\section{Safety and tolerability Medical review of potential adverse events}

Prior to medical review, 3.1\% $(n=97)$ of lasmiditan-treated patients and $1.4 \%(n=18)$ of placebo-treated patients were noted to have at least 1 potential $\mathrm{CV} \mathrm{AE}$, reported irrespective of time following dosage. Significant, although rare, reports of events were coded by SMQ as cardiomyopathy $(0.8 \%[n=25]$ of lasmiditan vs. $0.2 \%[n=2]$ of placebo $[\mathrm{OR}=5.08, p=0.01])$ and CNS vascular disorders $(0.3 \%$ $[n=11]$ of lasmiditan vs. $0.0 \%[n=0]$ of placebo $[p=0.03]$ ). Following medical review, $1.7 \%(n=55)$ of lasmiditantreated and $1.3 \%(n=16)$ of placebo-treated patients were considered to have at least 1 likely CV AE. A total of 44 cases were excluded (Additional file 1: Table S1), about half of which were due to abdominal pain with no other CV-related symptoms. Other examples of exclusion were due to events of shortness of breath or syncope (coded by SMQ as "cardiomyopathy") or dysarthria (coded by SMQ as "CNS vascular disorders") without having a history of these events, a comorbid cardiac event, or a concomitant $\mathrm{CV}$ medication. Details of the potential and likely CV AEs are presented in Additional file 1: Table S2 and Table S3, respectively, and are discussed in more detail in the Additional files.

\section{Likely CV treatment-emergent adverse events}

The number and percentage of patients with at least 1 likely CV TEAE, although not statistically significant, were higher in those treated with lasmiditan $(n=30$ [0.9\%]) than in those treated with placebo $(n=5$ [0.4\%]) (Table 3). In the Cardiac arrhythmias SMQ, a significantly greater number of events were reported 
Table 1 Summary of cardiovascular risk factors, other risk factors, and laboratory values and vital signs at baseline by sex

\begin{tabular}{|c|c|c|c|}
\hline Characteristic (unit) & $\begin{array}{l}\text { Females } \\
(N=3726) \\
n(\%)\end{array}$ & $\begin{array}{l}\text { Males } \\
(N=713) \\
n(\%)\end{array}$ & $\begin{array}{l}\text { Pooled } \\
(N=4439) \\
n(\%)\end{array}$ \\
\hline \multicolumn{4}{|l|}{ CVRFs per ACC/AHA recommended variables ${ }^{a}$} \\
\hline Age $>40$ years & $2044(54.9)$ & $387(54.3)$ & $2431(54.8)$ \\
\hline Current smoker & $490(13.2)$ & $139(19.5)$ & $629(14.2)$ \\
\hline High total cholesterol ( $\geq 240 \mathrm{mg} / \mathrm{dL})$ & $421(11.3)$ & $70(9.8)$ & $491(11.1)$ \\
\hline Low HDL cholesterol (< 40 mg/dL in men, $<50$ mg/dL in women) & $1197(32.1)$ & $206(28.9)$ & $1403(31.6)$ \\
\hline High blood pressure (SBP $\geq 140$ mmHg and/or medical history of hypertension at baseline) & $775(20.8)$ & $200(28.1)$ & $975(22.0)$ \\
\hline Medical history of diabetes mellitus, total & $215(5.8)$ & $53(7.4)$ & $268(6.0)$ \\
\hline Type 1 & $8(0.2)$ & $2(0.3)$ & $10(0.2)$ \\
\hline Type 2 & $154(4.1)$ & $31(4.3)$ & $185(4.2)$ \\
\hline Type unspecified & $53(1.4)$ & $20(2.8)$ & $73(1.6)$ \\
\hline \multicolumn{4}{|l|}{ Number of CVRFs } \\
\hline$\geq 1$ & 2939 (78.9) & $561(78.7)$ & $3500(78.8)$ \\
\hline$\geq 2$ & $1507(40.4)$ & $326(45.7)$ & $1833(41.3)$ \\
\hline$\geq 3$ & $545(14.6)$ & $125(17.5)$ & $670(15.1)$ \\
\hline$\geq 4$ & $133(3.6)$ & $36(5.0)$ & $169(3.8)$ \\
\hline$\geq 5$ & $18(0.5)$ & $7(1.0)$ & $25(0.6)$ \\
\hline$\geq 6$ & $0(0.0)$ & $0(0.0)$ & $0(0.0)$ \\
\hline \multicolumn{4}{|l|}{ Other risk factors of potential interest } \\
\hline Postmenopausal & $573(15.4)$ & N/A & N/A \\
\hline Obese (BMI $\geq 30 \mathrm{~kg} / \mathrm{m}^{2}$ ) & $1655(44.4)$ & $278(39.0)$ & $1933(43.5)$ \\
\hline History of migraine with aura & $1495(40.1)$ & $264(37.0)$ & 1759 (39.6) \\
\hline High LDL cholesterol ( $\geq 160$ mg/dL) & $245(6.6)$ & $46(6.5)$ & $291(6.6)$ \\
\hline Medical history of hypertension & $569(15.3)$ & $134(18.8)$ & $703(15.8)$ \\
\hline Family history of CAD & $1134(30.4)$ & $160(22.4)$ & $1294(29.2)$ \\
\hline \multicolumn{4}{|l|}{ Laboratory values and vital signs, mean (SD) } \\
\hline Total cholesterol (mg/dL) & $192.0(39.4)$ & $190.6(38.3)$ & $191.8(39.3)$ \\
\hline HDL cholesterol & $58.0(16.1)$ & $48.3(14.6)$ & $56.4(16.2)$ \\
\hline LDL cholesterol & $107.7(33.3)$ & $109.9(32.8)$ & $108.1(33.3)$ \\
\hline $\mathrm{SBP}(\mathrm{mm} \mathrm{Hg})$ & $120.0(13.9)$ & $127.5(12.9)$ & $121.2(14.0)$ \\
\hline
\end{tabular}

${ }^{\mathrm{a}} \mathrm{ACC} / \mathrm{AHA}$ guideline-recommended variables for CV risk assessment in adults without diagnosed disease [45]

ACC/AHA American College of Cardiology/American Heart Association, BMI Body mass index, CAD Coronary artery disease, CV Cardiovascular, CVRF Cardiovascular risk factor, $H D L$ High-density lipoprotein, $L D L$ Low-density lipoprotein, $N$ total number of patients in each group, $n$ number of patients with risk factor, $N / A$ Not applicable, SBP Systolic blood pressure, SD Standard deviation

in the lasmiditan-treated group, largely due to reports of palpitations, tachycardia, and increased heart rate. All events were mild to moderate.

There were no discontinuations due to likely CV TEAEs. No deaths were reported in any patients who took study drug or placebo. One lasmiditan-treated patient had a serious TEAE of worsening hypertension. The patient had preexisting hypertension, which was under treatment at screening with nifedipine 100 mg. The patient was hospitalized and symptoms resolved following an increase in nifedipine dose to 150 mg. The patient had normal blood pressure during screening $(120 / 84 \mathrm{mmHg})$ and at the end of study visit (110/74 mmHg).

\section{Likely CV treatment-emergent adverse events by CVRF categories}

Table 4 shows likely CV TEAEs when analyzed by the number of CVRFs. There was no statistical difference in the frequency of likely CV TEAEs either in the absence or presence of any CVRFs between the placebo and pooled lasmiditan treatment groups at either an SMQ or an individual PT level. The only likely CV TEAE seen across patients with $\geq 1, \geq 2, \geq 3$, or $\geq 4$ CVRFs was palpitations. No 
Table 2 Frequency of cardiovascular risk factors by dose

\begin{tabular}{llll} 
regimen & & \\
\hline Number of CVRFs $^{a}$ & Placebo & All LTN & Total \\
$N=1262$ & $N=3177$ & $N=4439$ \\
& $n(\%)$ & $n(\%)$ & $n(\%)$ \\
\hline 0 & $255(20.2)$ & $684(21.5)$ & $939(21.2)$ \\
1 & $486(38.5)$ & $1181(37.2)$ & $1667(37.6)$ \\
2 & $326(25.8)$ & $837(26.3)$ & $1163(26.2)$ \\
3 & $144(11.4)$ & $357(11.2)$ & $501(11.3)$ \\
4 & $43(3.4)$ & $101(3.2)$ & $144(3.2)$ \\
5 & $8(0.6)$ & $17(0.5)$ & $25(0.6)$ \\
\hline
\end{tabular}

${ }^{a}$ CVRFs were based on the American College of Cardiology/American Heart Association Task Force on Practice Guidelines [45] and included age, total and high-density lipoprotein cholesterol, systolic blood pressure (including treated or untreated status), diabetes, and current smoking status

CVRFs Cardiovascular risk factors, LTN Lasmiditan, $N$ number of patients in the analysis population, $n$ number of patients within each specific category

statistical dose response between the lasmiditan 100 and $200 \mathrm{mg}$ doses was observed for any of the likely CV TEAEs based on the CVRFs overall, with increasing number of risk factors, or for individual TEAEs. There were numerically more patients with a likely CV TEAE in the pooled lasmidi$\tan 200-\mathrm{mg}(n=14$ [1.1\%]) and 100-mg dose groups $(n=13$ [1.0\%]) compared with the 50-mg dose group $(n=3$ [0.5\%]). The 50-mg dose group was not included in the pooled test of trend because the 50-mg dose was included only in one of the studies.

\section{Likely CV adverse events and CV treatment-emergent adverse events by history of aura}

Given that aura is a potential factor that may increase the risk of CV events, likely CV AEs and TEAEs were summarized for patients with and without a history of aura for placebo and lasmiditan treatment groups. Likely CV AEs and likely CV TEAEs were similar regardless of history of aura (Additional file 1: Table S4 and Table S5, respectively) for both placebo- and lasmiditan-treated groups.

\section{Discussion}

Options for acute treatment of migraine attacks are limited in patients with prior history of $\mathrm{CV}$ and cerebrovascular diseases and CVRFs, which are a significant percentage of the migraine patient population especially with increasing age. Migraine itself is a risk factor for $\mathrm{CV}$ disease and $\mathrm{CV}$ events, and these associations are more significant in patients with aura. In addition, patients may have other risk factors including hypertension and diabetes. Finding a treatment that does not exacerbate these risks could improve safety over existing treatments such as triptans, which are contraindicated in patients with CV history or risk.

Lasmiditan is a centrally penetrant, non-vasoconstrictive, selective $5-\mathrm{HT}_{1 \mathrm{~F}}$ receptor agonist being developed for the acute treatment of migraine. Lasmiditan inhibits trigeminovascular nociception by activation of $5-\mathrm{HT}_{1 \mathrm{~F}}$ receptors [36]. The purpose of this analysis was to examine the safety and efficacy of lasmiditan in patients with CVRFs from two Phase 3 studies, SAMURAI and SPARTAN.

This pooled Phase 3 population included a wellbalanced population across lasmiditan- and placebotreated patients with respect to the presence of baseline CCRH (approximately 20\%) and 1 or more CVRFs (79\% with $\geq 1,41 \%$ with $\geq 2$, and $15 \%$ with $\geq 3$ CVRFs) in addition to their migraine history. The rates of risk factors appear to be generally

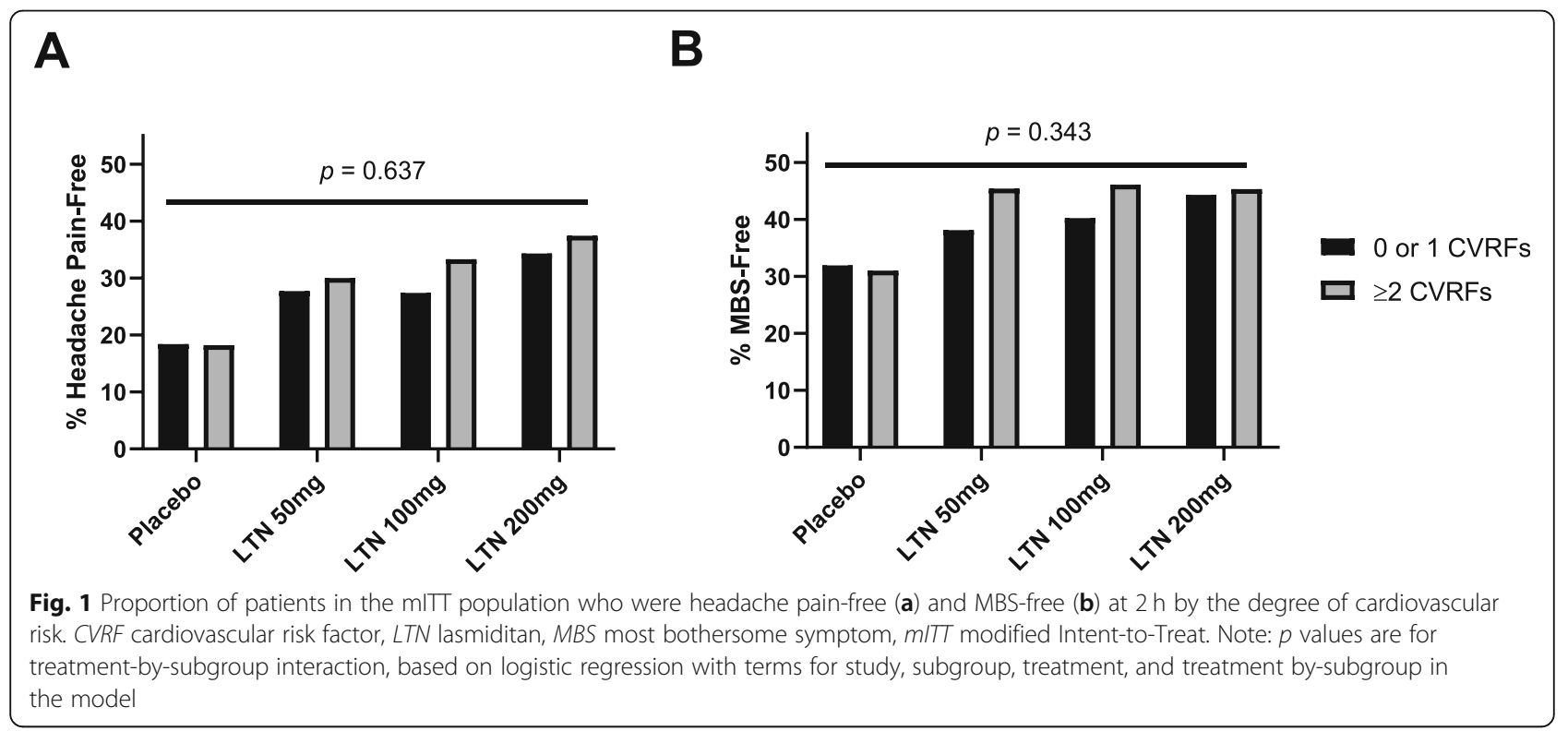


Table 3 Summary and analysis of likely cardiovascular treatment-emergent adverse events

\begin{tabular}{|c|c|c|c|c|c|}
\hline \multirow{2}{*}{$\begin{array}{l}\text { Standardized MedDRA Query } \\
\text { Preferred Term }\end{array}$} & \multirow{2}{*}{$\begin{array}{l}\text { Placebo } \\
(N=1262) \\
n(\%) \text { adj \%] }\end{array}$} & \multirow{2}{*}{$\begin{array}{l}\text { All LTN } \\
(N=3177) \\
n(\%) \text { [adj \%] }\end{array}$} & \multicolumn{3}{|c|}{ Comparison between all LTN vs. placebo } \\
\hline & & & $\overline{\mathrm{OR}^{a}}$ & $95 \% \mathrm{Cl}^{\mathrm{a}}$ & $p$ value $^{b}$ \\
\hline Patients with at least 1 likely CV TEAE & $5(0.4)[0.4]$ & $30(0.9)[0.9]$ & 2.46 & $(0.95,6.39)$ & 0.06 \\
\hline Cardiac arrhythmias (SMQ) & $3(0.2)[0.2]$ & $27(0.8)[0.9]$ & 3.59 & $(1.09,11.79)$ & 0.02 \\
\hline Palpitations & $1(0.1)[0.1]$ & $12(0.4)[0.4]$ & 4.67 & $(0.63,34.69)$ & 0.09 \\
\hline Tachycardia & $0(0.0)[0.0]$ & $6(0.2)[0.2]$ & & & 0.14 \\
\hline Heart rate increased & $1(0.1)[0.1]$ & $5(0.2)[0.2]$ & 1.89 & $(0.23,15.65)$ & 0.54 \\
\hline Bradycardia & $1(0.1)[0.1]$ & $1(0.0)[0.0]$ & 0.50 & & 0.62 \\
\hline Electrocardiogram abnormal & $0(0.0)[0.0]$ & $1(0.0)[0.0]$ & & & 0.48 \\
\hline Sinus bradycardia & $0(0.0)[0.0]$ & $1(0.0)[0.0]$ & & & 0.48 \\
\hline Syncope & $0(0.0)[0.0]$ & $1(0.0)[0.0]$ & & & 0.56 \\
\hline Cardiomyopathy (SMQ) & $1(0.1)[0.1]$ & $14(0.4)[0.4]$ & 5.45 & $(0.74,40.05)$ & 0.06 \\
\hline Palpitations & $1(0.1)[0.1]$ & $12(0.4)[0.4]$ & 4.67 & $(0.63,34.69)$ & 0.09 \\
\hline Electrocardiogram abnormal & $0(0.0)[0.0]$ & $1(0.0)[0.0]$ & & & 0.48 \\
\hline Syncope & $0(0.0)[0.0]$ & $1(0.0)[0.0]$ & & & 0.56 \\
\hline Hypertension (SMQ) & $0(0.0)[0.0]$ & $3(0.1)[0.1]$ & & & 0.28 \\
\hline Hypertension & $0(0.0)[0.0]$ & $2(0.1)[0.1]$ & & & 0.36 \\
\hline Blood pressure increased & $0(0.0)[0.0]$ & $1(0.0)[0.0]$ & & & 0.56 \\
\hline Pulmonary hypertension (SMQ) & $1(0.1)[0.1]$ & $0(0.0)[0.0]$ & 0.00 & & 0.16 \\
\hline Cardiac murmur & $1(0.1)[0.1]$ & $0(0.0)[0.0]$ & 0.00 & & 0.16 \\
\hline Torsade de pointes/QT prolongation (SMQ) & $0(0.0)[0.0]$ & $1(0.0)[0.0]$ & & & 0.56 \\
\hline Syncope & $0(0.0)[0.0]$ & $1(0.0)[0.0]$ & & & 0.56 \\
\hline Any abdominal pain (PT) & $1(0.1)[0.1]$ & $0(0.0)[0.0]$ & 0.00 & & 0.16 \\
\hline Abdominal pain upper & $1(0.1)[0.1]$ & $0(0.0)[0.0]$ & 0.00 & & 0.16 \\
\hline
\end{tabular}

${ }^{a}$ Mantel-Haenszel OR stratified by study and $95 \% \mathrm{Cl}(\mathrm{Cl}$ calculated if $\geq 4$ events in numerator and $\geq 1$ event in denominator)

${ }^{\mathrm{b}} p$ values are from Cochran-Mantel-Haenszel test of general association stratified by study. Bold indicates a $p$ value $<0.05$

adj \% study size adjusted percentage, CI Confidence interval, CNS Central nervous system, CV Cardiovascular, LTN Lasmiditan, MedDRA Medical Dictionary for Drug Regulatory Activities, $N$ Number of patients in the analysis population, $n$ number of patients within each specific category, OR Odds ratio, $P T$ Preferred Term, SMQ Standardized MedDRA Query, TEAE treatment-emergent adverse event

Likely CV TEAEs are from medical review out of potential CV TEAEs that are selected based on broad and narrow terms in the SMOs Cardiac arrhythmias, Cardiac failure, Cardiomyopathy, CNS vascular disorders, Embolic and thrombotic events, Hypertension, Ischemic heart disease, Pulmonary hypertension, and Torsade de pointes/QT prolongation and the PTs abdominal pain, abdominal pain upper, and abdominal pain lower

Any abdominal pain (PT) consists of the PTs abdominal pain, abdominal pain upper, and abdominal pain lower

MedDRA version 21.0

representative of the overall migraine population; for example, in the American Migraine Prevalence and Prevention study, patients with migraine with $\geq 1, \geq$ 2 , and $\geq 3$ risk factors are numbered $70 \%, 40 \%$, and $19 \%$, respectively [26].

Headache pain freedom and MBS freedom at $2 \mathrm{~h}$ examined by subgroups of 0 or 1 compared to $\geq 2$ CVRFs were not significantly different in any dose regimen of lasmiditan, indicating that lasmiditan efficacy is not affected by the presence of CVRFs. In general, a small number of CV AEs including TEAEs were reported in the placebo-controlled studies. There were no ischemic CV TEAEs reported. There was no statistical difference between placebo and lasmiditan in the frequency of likely CV TEAEs either in the absence or presence of CVRFs at either an SMQ or an individual PT level. The only likely CV TEAE seen across patients with $\geq 1$, $\geq 2, \geq 3$, or $\geq 4$ CVRFs was palpitations. The subjective $\mathrm{AE}$ descriptor palpitations (which includes tachycardia and increased heart rate) is considered an adverse drug reaction with lasmiditan; however, the reported incidence was $<1 \%$. Since concomitant vital signs or ECGs were not recorded during these symptoms, it is not known whether they were associated with true increases or decreases in heart rate. In clinical pharmacology studies using objective measures, lasmiditan was associated with decreases in heart rate of -5 to - 10 beats per minute following doses of 50 to $200 \mathrm{mg}$ (data unpublished). Additionally, the symptom of palpitations could reflect CV changes or actually wholly non-cardiac sources, such as anxiety or panic. In most of the cases, there was a report of other concurrent TEAEs, mostly neurological. Although the mechanism of action is unknown for which lasmiditan may cause palpitations or a 
Table 4 Summary and analysis of likely cardiovascular treatment-emergent adverse events by cardiovascular risk factor categories

\begin{tabular}{|c|c|c|c|c|c|}
\hline \multirow{2}{*}{$\begin{array}{l}\text { Categorical baseline CV risk } \\
\text { factors } \\
\text { Preferred Term }\end{array}$} & \multirow{2}{*}{$\begin{array}{l}\text { Placebo } \\
(N=1262) \\
n(\%) \text { adj \%] }\end{array}$} & \multirow{2}{*}{$\begin{array}{l}\text { All LTN } \\
(N=3177) \\
n(\%) \text { [adj \%] }\end{array}$} & \multicolumn{3}{|c|}{ Comparison between all LTN vs. placebo } \\
\hline & & & $\mathrm{OR}^{\mathrm{a}}$ & $95 \% \mathrm{Cl}^{\mathrm{a}}$ & $p$ value $^{b}$ \\
\hline 0 & $0(0.0)[0.0]$ & $5(0.2)[0.2]$ & & & 0.14 \\
\hline Bradycardia & $0(0.0)[0.0]$ & $1(0.0)[0.0]$ & & & 0.48 \\
\hline Hypertension & $0(0.0)[0.0]$ & $1(0.0)[0.0]$ & & & 0.56 \\
\hline Palpitations & $0(0.0)[0.0]$ & $1(0.0)[0.0]$ & & & 0.48 \\
\hline Sinus bradycardia & $0(0.0)[0.0]$ & $1(0.0)[0.0]$ & & & 0.48 \\
\hline Tachycardia & $0(0.0)[0.0]$ & $1(0.0)[0.0]$ & & & 0.56 \\
\hline$\geq 1$ & $5(0.4)[0.4]$ & $25(0.8)[0.8]$ & 2.03 & $(0.77,5.34)$ & 0.15 \\
\hline Palpitations & $1(0.1)[0.1]$ & $11(0.3)[0.3]$ & 4.22 & $(0.56,31.70)$ & 0.12 \\
\hline Heart rate increased & $1(0.1)[0.1]$ & $5(0.2)[0.2]$ & 1.89 & $(0.23,15.65)$ & 0.54 \\
\hline Tachycardia & $0(0.0)[0.0]$ & $5(0.2)[0.2]$ & & & 0.18 \\
\hline Blood pressure increased & $0(0.0)[0.0]$ & $1(0.0)[0.0]$ & & & 0.56 \\
\hline Electrocardiogram abnormal & $0(0.0)[0.0]$ & $1(0.0)[0.0]$ & & & 0.48 \\
\hline Hypertension & $0(0.0)[0.0]$ & $1(0.0)[0.0]$ & & & 0.48 \\
\hline Syncope & $0(0.0)[0.0]$ & $1(0.0)[0.0]$ & & & 0.56 \\
\hline Abdominal pain upper & $1(0.1)[0.1]$ & $0(0.0)[0.0]$ & 0.00 & & 0.16 \\
\hline Bradycardia & $1(0.1)[0.1]$ & $0(0.0)[0.0]$ & 0.00 & & 0.16 \\
\hline Cardiac murmur & $1(0.1)[0.1]$ & $0(0.0)[0.0]$ & 0.00 & & 0.16 \\
\hline$\geq 2$ & $3(0.2)[0.2]$ & $11(0.3)[0.3]$ & 1.48 & $(0.41,5.38)$ & 0.56 \\
\hline Palpitations & $0(0.0)[0.0]$ & $4(0.1)[0.1]$ & & & 0.20 \\
\hline Tachycardia & $0(0.0)[0.0]$ & $3(0.1)[0.1]$ & & & 0.28 \\
\hline Heart rate increased & $1(0.1)[0.1]$ & $2(0.1)[0.1]$ & 0.67 & & 0.74 \\
\hline Blood pressure increased & $0(0.0)[0.0]$ & $1(0.0)[0.0]$ & & & 0.56 \\
\hline Hypertension & $0(0.0)[0.0]$ & $1(0.0)[0.0]$ & & & 0.48 \\
\hline Abdominal pain upper & $1(0.1)[0.1]$ & $0(0.0)[0.0]$ & 0.00 & & 0.16 \\
\hline Cardiac murmur & $1(0.1)[0.1]$ & $0(0.0)[0.0]$ & 0.00 & & 0.16 \\
\hline$\geq 3$ & $0(0.0)[0.0]$ & $3(0.1)[0.1]$ & & & 0.28 \\
\hline Hypertension & $0(0.0)[0.0]$ & $1(0.0)[0.0]$ & & & 0.48 \\
\hline Palpitations & $0(0.0)[0.0]$ & $1(0.0)[0.0]$ & & & 0.56 \\
\hline Tachycardia & $0(0.0)[0.0]$ & $1(0.0)[0.0]$ & & & 0.56 \\
\hline$\geq 4$ & $0(0.0)[0.0]$ & $1(0.0)[0.0]$ & & & 0.56 \\
\hline Palpitations & $0(0.0)[0.0]$ & $1(0.0)[0.0]$ & & & 0.56 \\
\hline
\end{tabular}

${ }^{a}$ Mantel-Haenszel OR stratified by study and $95 \% \mathrm{Cl}(\mathrm{Cl}$ calculated if $\geq 4$ events in numerator and $\geq 1$ event in denominator)

${ }^{b} p$ values are from Cochran-Mantel-Haenszel test of general association stratified by study

ACC/AHA American College of Cardiology and American Heart Association, adj \% study size adjusted percentage, $\mathrm{Cl}$ confidence interval, CNS central nervous system, CV cardiovascular, HDL high-density lipoprotein, LTN lasmiditan, MedDRA Medical Dictionary for Drug Regulatory Activities, N number of patients in the analysis population, $n$ number of patients within each specific category, OR odds ratio, PT Preferred Term, SMQ Standardized MedDRA Query, TEAE treatmentemergent adverse event

Note: Likely CV TEAEs are from medical review out of potential CV TEAEs that are selected based on broad and narrow terms in the SMQs Cardiac arrhythmias, Cardiac failure, Cardiomyopathy, CNS vascular disorders, Embolic and thrombotic events, Hypertension, Ischemic heart disease, Pulmonary hypertension, and Torsade de pointes/QT prolongation and the PTs abdominal pain, abdominal pain upper, and abdominal pain lower

The CV disease risk factors are identified based on the ACC/AHA Task Force on Practice Guidelines [45]. A present/absent criterion was applied to each variable as follows: age $>40$ years for both men and women, diabetes mellitus (any), current smoker, total cholesterol $\geq 240 \mathrm{mg} / \mathrm{dL}$ (laboratory measure), $\mathrm{HDL}$ cholesterol $<40$ $\mathrm{mg} / \mathrm{dL}$ for men and $<50 \mathrm{mg} / \mathrm{dL}$ for women (laboratory measure), and systolic blood pressure $\geq 140 \mathrm{mmHg}$ (vital signs measure) and/or self-reported high blood pressure were included as hypertension

MedDRA version 21.0

decrease in heart rate, caution is advised for concomitant use of lasmiditan with other drugs that may lower heart rate.
Limitations of these analyses include the small sample size in patients with $\mathrm{CCRH}$, the single migraine attack design, the medical review of potential CV AEs was 
performed by unblinded Lilly physicians, and the lack of vital sign and ECG measurements around the time of dosing. Additionally, potential rare events require large sample sizes and longer duration of observation than was available in these single-attack studies. Despite the limitations, these studies provide insight into the efficacy and safety of lasmiditan in patients with CVRFs. Results of multiple-attack studies (such as NCT03670810 and NCT02565186) will provide insight into efficacy and safety over time.

\section{Conclusions}

We found that the proportion of patients achieving headache pain freedom and MBS freedom at $2 \mathrm{~h}$ were similar within subgroups of patients with 0 or 1 CVRF(s) and with $\geq 2$ CVRFs, indicating that lasmiditan efficacy is not affected by the presence of CVRFs. We also found no statistical difference between placebo and lasmiditan in the frequency of likely CV TEAEs either in the absence or presence of any CVRFs in these single-attack studies. The lack of differences in efficacy and safety with increasing numbers of CVRFs indicates that lasmiditan might be considered in the treatment algorithm for patients with CVRFs. Longer-term studies are needed to evaluate efficacy and safety over time.

\section{Additional file}

Additional file 1: Supplemental materials. Table S1. Summary of events excluded from likely adverse events and the associated Standardized MedDRA Queries/Preferred Terms. Table S2. Summary and analysis of potential cardiovascular adverse events within Standardized MedDRA Queries/Preferred Terms. Table S3. Summary and analysis of likely cardiovascular adverse events within Standardized MedDRA Queries/Preferred Terms. Table S4. Summary of likely cardiovascular adverse events within Standardized MedDRA Queries/Preferred Terms in patients with and without aura. Table S5. Summary of likely cardiovascular treatment-emergent adverse events within Standardized MedDRA Queries/Preferred Terms in patients with and without aura. (DOCX $39 \mathrm{~kb})$

\footnotetext{
Abbreviations

5- $\mathrm{HT}_{1 \mathrm{~B}}$ : 5-hydroxytrptamine receptor $1 \mathrm{~B} ; 5-\mathrm{HT}_{1 \mathrm{~F}}$ : 5-hydroxytryptamine receptor 1F; AE: Adverse event; aHR: adjusted hazard ratio; CAD: Coronary artery disease; $\mathrm{CCRH}$ : Cardiovascular/cerebrovascular-related history; Cl: Confidence interval; CNS: Central nervous system; CV: Cardiovascular; CVRF: Cardiovascular risk factor; ECG: Electrocardiogram; MBS: Most bothersome symptom; MedDRA: Medical Dictionary for Drug Regulatory Activities; OR: Odds ratio; PT: Preferred Term; SMQ: Standardized Medical Dictionary for Drug Regulatory Activities Query; TEAE: Treatment-emergent adverse event
}

\section{Acknowledgements}

Editorial and writing support was provided by Kristen P. Tolson, PhD, Samue C. Suarez, PhD, and Chastity Bradley, PhD (Synchrogenix, a Certara company).

\section{Authors' contributions}

EBD and RK were involved in study conception and design. HMH was responsible for acquisition of data. EBD, PHB, and RK performed data analysis. $\mathrm{RES}, \mathrm{HMH}, \mathrm{EBD}, \mathrm{RK}, \mathrm{EGD}, \mathrm{PHB}$, and AJS provided data interpretation. RES, $H M H, E B D, R K, E G D, P H B$, and AJS were involved in drafting/revising the article. RES, HMH, EBD, RK, EGD, PHB, and AJS provided final approval of the version to be published.

Authors' information

Not applicable.

Funding

These studies were funded by CoLucid Pharmaceuticals, Inc., a wholly owned subsidiary of Eli Lilly and Company.

\section{Availability of data and materials}

The datasets used and/or analyzed during the current study are available from the corresponding author upon reasonable request.

Ethics approval and consent to participate

These studies were conducted in accordance with the principles of the Declaration of Helsinki. The institutional review board or independent ethics committee at each site approved the protocols, and all patients provided written informed consent. All authors had access to the study data and have reviewed and approved the final manuscript.

\section{Consent for publication}

Not applicable.

\section{Competing interests}

RES was a Lilly-paid consultant for galcanezumab clinical trials Data Monitoring Committee. $\mathrm{HMH}, \mathrm{EBD}, \mathrm{RK}, \mathrm{EGD}$, and PHB are all employees and shareholders of Eli Lilly and Company and/or one of its subsidiaries. EGD was also an advisory board member for Eli Lilly prior to her employment and served on speakers' bureaus for Allergan Botox, Amgen, and Teva. AJS served as a consultant for Alder, eNeura, Amgen, Eli Lilly and Company, and Novartis. AJS also received grant funding from the Migraine Research Foundation Mayo Clinic Intramural funding.

\section{Author details}

${ }^{1}$ The University of Vermont Medical Center, 111 Colchester Ave, Burlington, VT 05401, USA. Eli Lilly and Company, and/or one of its subsidiaries, Lilly Corporate Center, Indianapolis, IN 46285, USA. ${ }^{3}$ Department of Psychological Sciences, Purdue University, 610 Purdue Mall, West Lafayette, IN 47907, USA. ${ }^{4}$ Mayo Clinic, 13400 E. Shea Blvd, Scottsdale, AZ 85259, USA.

Received: 30 May 2019 Accepted: 20 August 2019

Published online: 29 August 2019

\section{References}

1. Buse DC, Rupnow MF, Lipton RB (2009) Assessing and managing all aspects of migraine: migraine attacks, migraine-related functional impairment, common comorbidities, and quality of life. Mayo Clin Proc 84:422-435

2. Hawkins K, Wang S, Rupnow MF (2007) Indirect cost burden of migraine in the United States. J Occup Environ Med 49:368-374

3. GBD 2016 Disease and Injury Incidence and Prevalence Collaborators (2017) Global, regional, and national incidence, prevalence, and years lived with disability for 328 diseases and injuries for 195 countries, 1990-2016: a systematic analysis for the Global Burden of Disease Study 2016. Lancet 390:1211-1259

4. Hippisley-Cox J, Coupland C, Brindle P (2017) Development and validation of QRISK3 risk prediction algorithms to estimate future risk of cardiovascular disease: prospective cohort study. BMJ 357:j2099

5. Mahmoud AN, Mentias A, Elgendy AY, Qazi A, Barakat AF, Saad M et al (2018) Migraine and the risk of cardiovascular and cerebrovascular events: a meta-analysis of 16 cohort studies including 1152407 subjects. BMJ Open 8:e020498

6. Kurth T, Winter AC, Eliassen AH, Dushkes R, Mukamal KJ, Rimm EB et al (2016) Migraine and risk of cardiovascular disease in women: prospective cohort study. BMJ 353:i2610

7. Kurth T, Gaziano JM, Cook NR, Logroscino G, Diener HC, Buring JE (2006) Migraine and risk of cardiovascular disease in women. JAMA 296:283-291

8. Becker C, Brobert GP, Almqvist PM, Johansson S, Jick SS, Meier CR (2007) Migraine and the risk of stroke, TIA, or death in the UK (CME). Headache 47:1374-1384 
9. Sacco S, Ornello R, Ripa P, Tiseo C, Degan D, Pistoia F et al (2015) Migraine and risk of ischaemic heart disease: a systematic review and meta-analysis of observational studies. Eur J Neurol 22:1001-1011

10. Peng KP, Chen YT, Fuh JL, Tang CH, Wang SJ (2017) Migraine and incidence of ischemic stroke: a nationwide population-based study. Cephalalgia 37:327-335

11. Adelborg K, Szepligeti SK, Holland-Bill L, Ehrenstein V, Horváth-Puhó E, Henderson WW et al (2018) Migraine and risk of cardiovascular diseases: Danish population based matched cohort study. BMJ 360:k96

12. Bigal ME, Kurth T, Santanello N, Buse D, Golden W, Robbins M et al (2010) Migraine and cardiovascular disease: a population-based study. Neurology 74:628-635

13. Etminan M, Takkouche B, Isorna FC, Samii A (2005) Risk of ischaemic stroke in people with migraine: systematic review and meta-analysis of observational studies. BMJ 330:63

14. Silberstein SD (2000) Practice parameter: evidence-based guidelines for migraine headache (an evidence-based review): report of the Quality Standards Subcommittee of the American Academy of Neurology. Neurology 55:754-762

15. Marmura MJ, Silberstein SD, Schwedt TJ (2015) The acute treatment of migraine in adults: the American Headache Society evidence assessment of migraine pharmacotherapies. Headache 55:3-20

16. FDA. FDA Drug Safety Communication: FDA strengthens warning that nonaspirin nonsteroidal anti-inflammatory drugs (NSAIDs) can cause heart attacks or strokes, 2015. https://www.fda.gov/Drugs/DrugSafety/ucm451800. htm. Accessed 25 Apr 2019

17. Coxib and Traditional NSAID Trialists' (CNT) Collaboration, Bhala N, Emberson J, Merhi A, Abramson S, Arber N et al (2013) Vascular and upper gastrointestinal effects of non-steroidal anti-inflammatory drugs: meta-analyses of individual participant data from randomised trials. Lancet 382:769-779

18. Bally M, Dendukuri N, Rich B, Nadeau L, Helin-Salmivaara A, Garbe E et al (2017) Risk of acute myocardial infarction with NSAIDs in real world use: Bayesian meta-analysis of individual patient data. BMJ 357:j1909

19. Imitrex tablets prescribing information, 2012. GlaxoSmithKline Research Triangle Park, NC. http://us.gsk.com/products/assets/us_imitrex_tablets.pdf . Accessed 25 Apr 2019

20. Amerge (naratriptan hydrochloride) tablets prescribing information, 2016. GlaxoSmithKline Research Triangle Park, NC. https://www.gsksource.com/ pharma/content/dam/GlaxoSmithKline/US/en/Prescribing_Information/ Amerge/pdf/AMERGE-PI-PIL.PDF . Accessed 25 Apr 2019

21. Maxalt (rizatriptan benzoate) prescribing information, 2013. Merck \& Co. Whitehouse Station, NJ. https://www.merck.com/product/usa/pi_circulars/ m/maxalt/maxalt_pi.pdf . Accessed 25 Apr 2019

22. Relpax (eletriptan hydrobromide) tablets prescribing information, 2008 Roerig division of Pfizer New York, NY. http://dailymed.nlm.nih.gov/ dailymed/lookup.cfm?setid=986dc112-b97-44a3-bfaf-074f906f8bb2 . Accessed 25 Apr 2019

23. Zomig (zolmitriptan) prescribing information, 2012. AstraZeneca/Impax Pharmaceuticals Hayward, CA. http://dailymed.nlm.nih.gov/dailymed/lookup. cfm?setid=294d60f3-005b-40d9-a404-cdd77f62b820. Accessed 25 Apr 2019

24. Frova (frovatriptan succinate) prescribing information, 2007. Endo Pharmaceuticals, Inc. Chadds Ford, PA. http://dailymed.nIm.nih.gov/ dailymed/lookup.cfm?setid=294d60f3-005b-40d9-a404-cdd77f62b820 Accessed 25 Apr 2019

25. Axert (almotriptan malate) tablets prescribing information, 2011; Janssen Pharmaceuticals, Inc. Titusville, NJ. http://dailymed.nlm.nih.gov/dailymed/lookup. cfm?setid=b13ba9bf-1661-456a-8eae-6d110ea08c94 . Accessed 25 Apr 2019

26. Buse DC, Reed ML, Fanning KM, Kurth T, Lipton RB (2017) Cardiovascular events, conditions, and procedures among people with episodic migraine in the US population: results from the American Migraine Prevalence and Prevention (AMPP) study. Headache 57:31-44

27. Goadsby PJ (2009) The vascular theory of migraine--a great story wrecked by the facts. Brain 132:6-7

28. Neeb L, Meents J, Reuter U (2010) 5-HT (1F) receptor agonists: a new treatment option for migraine attacks? Neurotherapeutics 7:176-182

29. Xavier AS, Lakshmanan M, Gunaseelan V (2017) The journey of the non-vascular relief for migraine: from 'triptans' to 'ditans'. Curr Clin Pharmacol 12:36-40

30. Goldstein DJ, Roon Kl, Offen WW, Ramadan NM, Phebus LA, Johnson KW et al (2001) Selective seratonin 1F (5-HT (1F)) receptor agonist LY334370 for acute migraine: a randomised controlled trial. Lancet 358:1230-1234
31. Johnson KW, Phebus LA, Cohen ML (1998) Serotonin in migraine: theories, animal models and emerging therapies. Prog Drug Res 51:219-244

32. Johnson KW, Schaus JM, Durkin MM, Audia JE, Kaldor SW, Flaugh ME et al (1997) 5-HT1F receptor agonists inhibit neurogenic dural inflammation in guinea pigs. Neuroreport 8:2237-2240

33. Ramadan NM, Skljarevski V, Phebus LA, Johnson KW (2003) 5-HT1F receptor agonists in acute migraine treatment: a hypothesis. Cephalalgia 23:776-785

34. Vila-Pueyo M (2018) Targeted 5-HT1F therapies for migraine. Neurotherapeutics 15:291-303

35. Nelson DL, Phebus LA, Johnson KW, Wainscott DB, Cohen ML, Calligaro DO et al (2010) Preclinical pharmacological profile of the selective 5-HT1F receptor agonist lasmiditan. Cephalalgia 30:1159-1169

36. Vila-Pueyo M, Strother IC, Page K, Loaraine H, Kovalchin J, Goadsby PJ et al (2016) Lasmiditan inhibits trigeminovascular nociceptive transmission. Cephalalgia 36(Suppl 1):152-153

37. Nilsson T, Longmore J, Shaw D, Pantev E, Bard JA, Branchek T et al (1999) Characterisation of $5-\mathrm{HT}$ receptors in human coronary arteries by molecular and pharmacological techniques. Eur J Pharmacol 372:49-56

38. Rubio-Beltran E, Labastida-Ramirez A, Villalon CM, MaassenVanDenBrink A (2018) Is selective 5-HT1F receptor agonism an entity apart from that of the triptans in antimigraine therapy? Pharmacol Ther 186:88-97

39. Razzaque Z, Heald MA, Pickard JD, Maskell L, Beer MS, Hill RG et al (1999) Vasoconstriction in human isolated middle meningeal arteries: determining the contribution of 5-HT1B- and 5-HT1F-receptor activation. Br J Clin Pharmacol 47:75-82

40. Bouchelet I, Case B, Olivier A, Hamel E (2000) No contractile effect for 5 HT1D and 5-HT1F receptor agonists in human and bovine cerebral arteries: similarity with human coronary artery. Br J Pharmacol 129:501-508

41. Rubio-Beltrán E, Haanes K, Labastida-Ramírez A, de Vries R, Danser J, Gralinski M et al (2016) Lasmiditan and sumatriptan: comparison of in vivo vascular constriction in the dog and in vitro contraction of human arteries. Cephalalgia 36(1S):104-105

42. Rubio-Beltrán E, Labastida-Ramírez A, van den Bogaerdt A, Bogers A, Zanelli $E$, Meeus $L$ et al (2017) In vitro characterization of agonist binding and functional activity at a panel of serotonin receptor subtypes for lasmiditan, triptans and other 5-HT receptor ligands and activity relationships for contraction of human isolated coronary artery. Cephalalgia 37(1S):363

43. Kuca B, Silberstein SD, Wietecha L, Berg PH, Dozier G, Lipton RB et al (2018) Lasmiditan is an effective acute treatment for migraine: a phase 3 randomized study. Neurology. 91(24):e2222-e2232

44. Goadsby PJ, Wietecha LA, Dennehy EB, Kuca B, Case MG, Aurora SK et al (2019) Phase 3 randomized, placebo-controlled, double blind study of lasmiditan for acute treatment of migraine. Brain 142:1894-1904

45. Goff DC Jr, Lloyd-Jones DM, Bennett G, Coady S, D'Agostino RB, Gibbons R et al (2014) 2013 ACC/AHA guideline on the assessment of cardiovascular risk: a report of the American College of Cardiology/American Heart Association Task Force on Practice Guidelines. Circulation 129:S49-S73

46. Expert Panel on Detection, Evaluation, and Treatment of High Blood Cholesterol in Adults (2001) Executive summary of the third report of The National Cholesterol Education Program (NCEP) expert panel on detection, evaluation, and treatment of high blood cholesterol in adults (Adult Treatment Panel III). JAMA 285:2486-2497

47. Whelton PK, Carey RM, Aronow WS, Casey DE Jr, Collins KJ, Dennison Himmelfarb C et al (2018) 2017 ACC/AHA/AAPA/ABC/ACPM/AGS/APhA/ ASH/ASPC/NMA/PCNA guideline for the prevention, detection, evaluation, and management of high blood pressure in adults: a report of the American College of Cardiology/American Heart Association Task Force on Clinical Practice Guidelines. J Am Coll Cardiol 71:e127-e248

48. Crowe B, Chuang-Stein C, Lettis S, Brueckner A (2016) Reporting adverse drug reactions in product labels. Ther Innov Regul Sci 50:455-463

\section{Publisher's Note}

Springer Nature remains neutral with regard to jurisdictional claims in published maps and institutional affiliations. 